Vietnam Journal of Mechanics; VAST, Vol. 26, 2004, No.3 (167-181)

\title{
EFFECTS OF SHEAR DEFORMATION ON LARGE DEFLECTION BEHAVIOR OF ELASTIC FRAMES
}

\author{
NGUYEN DINH KIEN \\ Institute of Mechanics
}

\begin{abstract}
In this paper, the effects of shear deformation on the large deflection behavior of elastic frames is investigated by the finite element method. A two-node nonlinear beam element with the shear deformation is formulated and employed to analyze some frame structures. The element based on the energy method is developed in the context of the corotational approach. A bracketing procedure used the lowest eigenvalue of structural tangent stiffness matrix as indicating parameter is adopted to compute the critical loads. An incremental/iterative procedure with the arc-length control technique is employed to trace the equilibrium paths. The numerical results show that the shear deformation plays an important role in the critical load and the large deflection behavior of the frames constructed from the components having low slenderness. A detail investigation is carried out to highlight the influence of slenderness on the behavior of the frames under large deflection.
\end{abstract}

\section{Introduction}

With the increment of using high performance material in practice, the structures can undergo large deformation before failure, and this phenomenon accelerates the importance of nonlinear analysis in the field of structural mechanics. It is well known that the finite element method is an effective tool in solving nonlinear problems of structures, and some commercial finite element programs, including MARC, SAP, ANSYS, ABAQUS and ADINA, have incorporated the nonlinear elements to make them possible for nonlinear analysis [1]

In the previous works $[2,3]$, the author and his co-worker have formulated some nonlinear beam elements for large deflection analysis of elastic frame structures. The beam elements developed in the work are based on the Euler-Bernoulli beam theory, in which a plane initially normal to the beam neutral axis remains plane and normal to this axis after deformation. This assumption ignores the shear deformation effects, which is valid for slender beams or frames constructed from slender components. As a consequence, the elements show a good performance in modelling the large deflection behavior of the slender frame structures.

In recent work [4], the author investigated the influence of shear deformation on the critical load and the post-buckling behavior of some elastic beams resting on a twoparameter elastic foundation. The investigation shows that the shear deformation plays an important role on the critical load as well as the post-buckling behavior of the beams. Taking the shear deformation into account, a considerable reduction in the critical load 


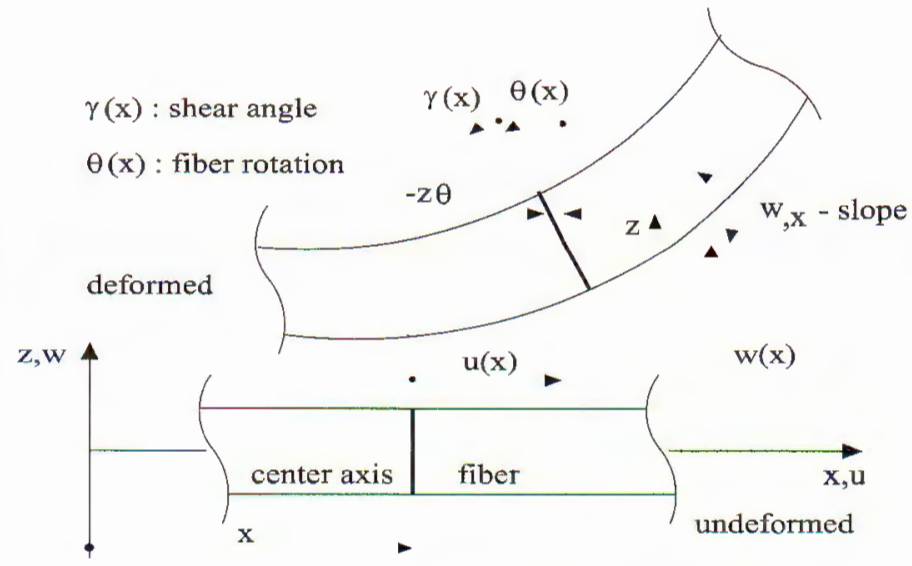

Fig. 1. Kinematics of Timoshenko beam theory

and the post-buckling strength of the beams has been observed. The numerical results obtained in [4]convince the author to perform more investigation on the effects of shear deformation on the large deflection bchavior of elastic frame structures, and the present paper aims to discuss this topic.

To investigate the shear deformation effects on the large deflection behavior of the irame structures by the finite element method, a nonlinear beam element enable to model the shear deformation should be employed. Naturally, the beam element based on the total Lagrange formulation presented in $[2,3]$ can be adopted for the purpose. However, as shown in, the beam elements formulated from the co-rotational approach exhibit some advantages comparing the total Lagrange formulation based counterparts, such as the simplicity and the ability in modelling the large rotation. Therefore, the approach is therefore adopted again in the present work to formulate the nonlinear beam element with shear deformation.

The paper is organized as follows: Section 2 gives a brief discussion on the Timoshenko beam theory. The finite element formulations are presented in Section 4. Section 4 discusses the numerical algorithm needed in computing the critical loads and the equilibrium paths. The numerical investigations are presented in Section 5. Finally, the main conclusions of the paper are summarized in Section 6.

\section{Timoshenko beam theory}

The Euler-Bernoulli beam theory ignored the shear deformation effects can be employed to analyze the slender beams and frames. For short stubby beams, the contribution of the shear deformation clearly cannot be neglected, and the Timoshenko beam theory should be employed instead of. The main points of the Timoshenko beam theory for $2 \mathrm{D}$ beams are summarized below.

The Timoshenko beam theory is based on the assumption that a plane initially normal to center axis of the beam remains plane, but not necessarily normal to the axis after deformation. In Fig. 1, $\theta$ represents the rotation of a fiber that was initially normal to the 
undeformed center axis. The total slope $\partial w / \partial x$ of the center axis is stemming respectively from the bending and the shear deformation as

$$
\frac{\partial w}{\partial x}=\theta(x)+\gamma(x)
$$

where $\theta(x)$ is the rotation of line elements along the center axis due to bending only; $\gamma(x)$ is the shear angle, and it equals to zero in the Euler-Bernoulli beam theory. The displacement field is given by

$$
\begin{aligned}
& U_{1}(x, y, z)=u(x)-z \theta(x)=u(x)-z\left[\frac{\partial w}{\partial x}-\gamma(x)\right], \\
& U_{2}(x, y, z)=0 \\
& U_{3}(x, y, z)=w(x),
\end{aligned}
$$

where $u(x), w(x)$ are the axial and transversal displacements of the point on the center axis; $z$ is the distance from the considering point to the center axis (see Fig. 1). With the linearly elastic assumption, the strains and stresses follow from Eqs.(2.2) are of the form

$$
\begin{aligned}
\epsilon_{x x} & =\frac{\partial u}{\partial x}-z \frac{\partial \theta}{\partial x}=\epsilon_{0}-z \kappa & \epsilon_{x z} & =\frac{1}{2} \gamma(x), \\
\sigma_{x x} & =E \epsilon_{x x}, & \tau_{x z} & =G \gamma(x),
\end{aligned}
$$

with $E$ and $G$ are the Young and shear modulus, respectively; $\kappa$ is the beam curvature. The shear stress given by Eq.(2.3b) is, however, incorrect due to its constant distribution through the beam thickness. In fact, the shear stress distribution though the thickness is quadratic, and to account for this matter, a shear correction factor $\psi$ is introduced, so that $[5]$

$$
\tau_{x z}=\psi G \gamma(x)
$$

The value of $\psi$ depends on the geometry of the beam cross-section. For a rectangular cross-section $\psi=\frac{10(1+\nu)}{12+11 \nu}$.

The strain energy of a beam element with length $l$ can easily formulated from Eqs.(2.3) and (2.4) as

$$
U=\frac{1}{2} \int_{0}^{l}\left(E A \epsilon_{0}^{2}+E I \kappa^{2}+\psi G A \gamma^{2}\right) d x
$$

where $A$ is the cross-sectional area of the beam; EA,EI, GA are the axial, bending and shear rigidities, respectively. The linear finite element formulation based on the Timoshenko beam theory is called Mindlin beam element [6], or Reissner-Mindlin beam element $[7]$.

A difference method to account for the non-constant distribution of the shear stress through the beam thickness is to add a third-order term for the axial displacement defined in Eq. (2.2a), and the corresponding theory is named 'third-order term theory' [8]. The third-order term theory captures the true variation of the transverse shear stress, and does not need the shear correction factor. However, the expressions for element developed from the third-order term theory are lengthy and complex, and the theory is not considered in the present work. 


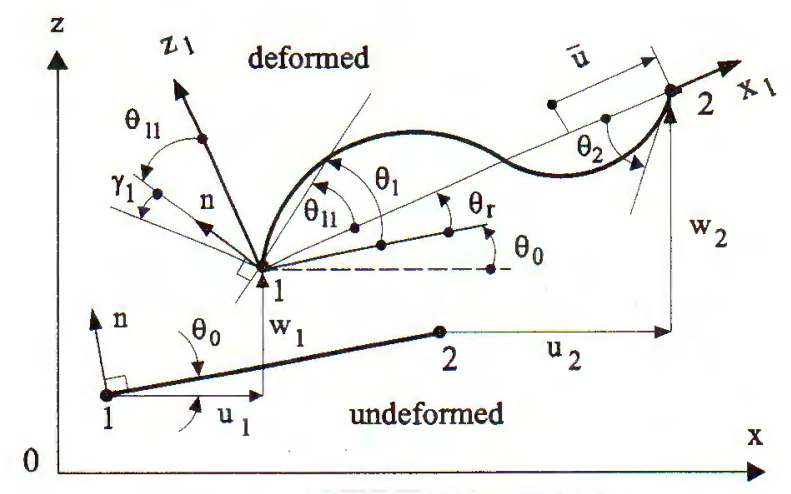

Fig. 2. Co-rotational beam element based on Timoshenko theory

\section{Beam element with shear deformation}

A two-node nonlinear beam element based on the Timoshenko beam theory is formulated in this section by employing the co-rotational approach. Fig. 2 shows the undeformed and deformed configurations of the beam element initially inclined an angle $\theta_{0}$ to the horizontal direction. The beam kinematics are defined in two coordinate systems, the local $\left(x_{l}, z_{l}\right)$ and the global $(x, z)$ ones. The local system moves and rotates with the element during its deformation process, and its original is always placed at node 1 and the $x_{l}$ axis directs to node 2. Accordingly, the vector of nodal displacements in the local and global systems are respectively given by

$$
\begin{aligned}
\mathbf{d}_{l} & =\left\{\begin{array}{llll}
u_{l 2} & \theta_{l 1} & \theta_{l 2}
\end{array}\right\}^{T}=\left\{\begin{array}{llll}
\bar{u} & \theta_{l 1} & \theta_{l 2}
\end{array}\right\}^{T}, \\
\mathbf{d} & =\left\{\begin{array}{llllll}
u_{1} & w_{1} & \theta_{1} & u_{2} & w_{2} & \theta_{2}
\end{array}\right\}^{T},
\end{aligned}
$$

where $(\ldots)^{T}$ denotes the transpose of a vector or a matrix; ' $l$ ' stands for 'local'. The relationships between the local and global nodal displacements in Eq.(3.1) are obtained from geometry consideration as

$$
\begin{aligned}
\bar{u} & =l_{n}-l, \\
\theta_{l 1} & =\theta_{1}-\theta_{r}, \\
\theta_{l 2} & =\theta_{2}-\theta_{r},
\end{aligned}
$$

where $l$ and $l_{n}$ are the initial and current length of the element; $\theta_{r}$ is the rigid rotation. $l, l_{n}, \theta_{r}$ can easily be expressed through the global nodal displacements .

The vectors of local and global nodal forces corresponding to the global and local vectors defined in Eqs. (3.1a) and (3.1b) are given by

$$
\begin{aligned}
\mathbf{f}_{l} & =\left\{\begin{array}{llll}
N_{l} & M_{l 1} & M_{l 2}
\end{array}\right\}^{T}, \\
\mathbf{f} & =\left\{\begin{array}{llllll}
N_{1} & V_{1} & M_{1} & N_{2} & V_{2} & M_{2}
\end{array}\right\}^{T},
\end{aligned}
$$

where $N_{i}, V_{i}, M_{i}, \quad(i=1,2)$ denote the global axial, transversal loads, and moment at node $i$, respectively; The global nodal force can be derived from the strain energy 
expression, which is invariant to the coordinate systems

$$
\mathbf{f}=\frac{\partial U}{\partial \mathbf{d}}=\frac{\partial U}{\partial \mathbf{d}_{1}} \frac{\partial \mathbf{d}_{1}}{\partial \mathbf{d}}=\mathbf{T}_{1}^{T} \mathbf{f}_{l}
$$

where $\mathbf{f}_{l}$ is the local nodal force vector; $\underset{(3 \times 6)}{\mathbf{T}_{1}}=\left[\frac{\partial \mathbf{d}_{l}}{\partial \mathbf{d}}\right]$ is the transformation matrix, which can easily be computed from Eqs.(3.2). The element tangent stiffness matrix can be formulated as differentiation of the nodal force vector (3.4) with respective to the global nodal displacements as

$$
\mathbf{k}_{t}=\frac{\partial \mathbf{f}}{\partial \mathbf{d}}=\mathbf{T}_{1}^{T} \mathbf{k}_{t l} \mathbf{T}_{1}+\mathbf{T}_{2} N_{l}+\mathbf{T}_{3}\left(M_{l 1}+M_{l 2}\right)
$$

where $\underset{(3 \times 3)}{\mathbf{k}_{t l}}=\left[\frac{\partial \mathbf{f}_{l}}{\partial \mathbf{d}_{l}}\right]$ is the local tangent stiffness matrix; $\underset{(6 \times 6)}{\mathbf{T}_{2}}=\left[\frac{\partial^{2} \bar{u}}{\partial \mathbf{d}^{2}}\right]$, and $\underset{(6 \times 6)}{\mathbf{T}_{3}}=$ $\left[\frac{\partial^{2} \theta_{l 1}}{\partial \mathrm{d}^{2}}\right]=\left[\frac{\partial^{2} \theta_{l 2}}{\partial \mathbf{d}^{2}}\right]=-\left[\frac{\partial^{2} \theta_{r}}{\partial \mathbf{d}^{2}}\right]$ are the transformation matrices, which can also be derived from the local and global relationship, Eqs. (3.2). With the aid of Eqs. (3.4) and (3.5), the remain work for obtaining the element formulations is to formulate the nodal force vector and tangent stiffness matrix in the local system, and these quantities are derived below.

Since the shear deformation is taken into account, the transverse displacement $w$ and the rotation $\theta$ are independent parameters. Accordingy, the linear functions can be employed to interpolate the displacement field for Timoshenko beam element $[9,10]$

In this regard, the local displacements and the shear strain are expressed through the local nodal displacement as

$$
\begin{gathered}
u_{l}=\frac{x_{l}}{l} \bar{u}, \quad \theta_{l}=\frac{l-x_{l}}{l} \theta_{l 1}+\frac{x_{l}}{l} \theta_{l 2} \\
\gamma_{l}=\frac{\partial w}{\partial x_{l}}-\theta_{l}=-\left(\frac{l-x_{l}}{l} \theta_{l 1}+\frac{x_{l}}{l} \theta_{l 2}\right),
\end{gathered}
$$

From Eq. (3.6) and Eqs. (2.3),(2.5), where the kinematics are understood as measures in the local system, one can write the strain energy in terms of local nodal displacements as

$$
U=\frac{1}{2} \int_{0}^{l}\left[E A\left(\frac{\bar{u}}{l}\right)^{2}+E I\left(\frac{\theta_{l 2}-\theta_{l 1}}{l}\right)^{2}+\psi G A\left(\frac{l-x_{l}}{l} \theta_{l 1}+\frac{x_{l}}{l} \theta_{l 2}\right)^{2}\right] d x_{l}
$$

The finite element formulation formulated from the strain energy defined by Eq. (3.7) is too stiff and leads to the shear locking problem $[8,9]$. To overcome the problem, the socalled reduced-integration technique, one-point Gauss quadrature for the present problem is employed to evaluate the strain energy expression, Eq. (3.7). In this regard, one can write Eq. (3.7) in a simple form

$$
U=\frac{1}{2 l} E A \bar{u}^{2}+\frac{1}{2 l} E I\left(\theta_{l 2}-\theta_{l 1}\right)^{2}+\frac{l}{8} \psi G A\left(\theta_{l 2}+\theta_{l 1}\right)^{2},
$$


The components of the local internal nodal force vector defined in Eq. (3.3a) are easily derived from Eq. (3.8) as

$$
\begin{aligned}
N_{l} & =\frac{1}{l} E A \bar{u}, \\
M_{l 1} & =\frac{1}{l} E I\left(\theta_{l 1}-\theta_{l 2}\right)+\frac{1}{4} \psi l G A\left(\theta_{l 1}+\theta_{l 2}\right), \\
M_{l 2} & =\frac{1}{l} E I\left(\theta_{l 2}-\theta_{l 1}\right)+\frac{1}{4} \psi l G A\left(\theta_{l 1}+\theta_{l 2}\right),
\end{aligned}
$$

The local tangent stiffness matrix is obtained by differentiation of the local nodal force vector, and given by

$$
\mathbf{k}_{t l}=\left[\begin{array}{ccc}
\frac{1}{l} E A & 0 & 0 \\
0 & \frac{1}{l} E I+\frac{1}{4} \psi l G A & -\frac{1}{l} E I+\frac{1}{4} \psi l G A \\
0 & -\frac{1}{l} E I+\frac{1}{4} \psi l G A & \frac{1}{l} E I+\frac{1}{4} \psi l G A
\end{array}\right],
$$

Eqs. (3.9), (3.10) combining with Eqs. (3.4) and (3.5) give the expressions for the global internal nodal force vector and tangent stiffness matrix of the element, thus completely define the finite element formulations. The Timoshenko element formulated in this paper is denoted ' $b 2 T i$ '.

\section{Numerical algorithm}

The equilibrium equations for non-linear analysis of structures are obtained by setting the out-of-balance forces to zeros $[1,11]$

$$
\mathbf{g}(\mathbf{p}, \lambda)=\mathbf{q}_{i}(\mathbf{p})-\lambda \mathbf{f}_{e f}=\mathbf{0},
$$

where the out-of-balance force vector $\mathbf{g}$ is a function of the current structural nodal displacements $\mathbf{p}$, and the 'load-level parameter' $\lambda ; \mathbf{q}_{i}$ is the structural internal force vector, obtained by assembling the previously developed vector $\mathbf{f}$ of each element; $\mathbf{f}_{\mathrm{e} f}$ is a 'fixed external loading vector'. 'The non-linear equations (4.1) can be solved by the so-called incremental/iterative technique based on the Newton-Rapson method, in which the norm of vector $\mathbf{g}(\mathbf{p}, \lambda)$ is guided towards zero.

To compute critical loads, the indirect method discussed by Shi in [12] and Crisfield in [13] is adopted herewith. The method has been implemented by the author using the lowest eigenvalue of the structural tangent stiffness matrix as indicating parameter [4] . For completeness, the main ideas of the method are illustrated in Fig. 3 and summarized below.

The lowest eigenvalue of structural tangent $\mathbf{K}_{t}$, merging from the element stiffness $\mathbf{k}_{t}$ formulated in Section 3, changes its sign when passing a singular point, a bifurcation or a limit point. Using a load control strategy in solving the equilibrium equations, Eqs. (4.1), for a 'perfect' structure (without any imperfection), the lowest eigenvalue of $\mathbf{K}_{t}$ is monitored at each load increment. When passing the lowest bifurcation point, predicted by the negative sign of $\tau[12]$, the bracketing procedure is active to compute an 

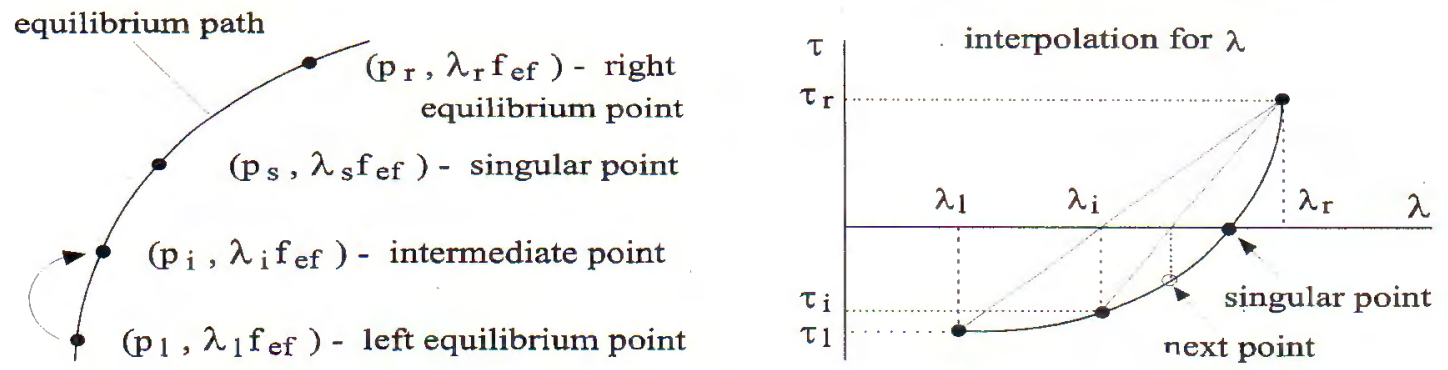

Fig. 3. Bracketing procedure for computing a singular point

intermediate point $\lambda_{i}$ [13], by using an interpolation scheme for the control parameter $\lambda$ as illustrated in Fig. 3. The bracketing algorithm contains the following steps:

1. Compute intermediate value $\lambda_{i}$ using the interpolation scheme as

$$
\lambda_{i}=\lambda_{l}-\frac{\lambda_{r}-\lambda_{l}}{\tau_{r}-\tau_{l}} \tau_{l}
$$

where $\lambda_{l}, \lambda_{r}$ are the values of the load-level parameter at equilibrium points on the 'left' and 'right' sides of the bifurcation point (before and after passing the bifurcation point), respectively; $\tau_{l}$ and $\tau_{r}$ are the lowest eigenvalues of $\mathbf{K}_{t}$ at the points.

2. Check convergency criteria, using $[4,13]$

$$
\left(\left|\frac{\lambda_{r}-\lambda_{l}}{\lambda_{i}} \bar{\tau}\right|\right)^{1 / 2}<\beta \text {, with } \bar{\tau}=\frac{\tau_{i}}{\left(\left|\tau_{l} \tau_{r}\right|\right)^{1 / 2}}
$$

where $\beta$ is a tolerance factor, taken by $10^{-4}$ in all the numerical studies in Sec. 5 . The obtained value $\lambda_{i}$ from the bracketing procedure is taken as the critical loadlevel parameter. Stop if the convergency criteria satisfies. If not, proceed to step 3.

3. Compute intermediate displacements $\mathbf{p}_{i}$ corresponding $\lambda_{i}$ and then the lowest eigenvalue $\tau_{i}$ of $\mathbf{K}_{t}$ at the intermediate point $\left(\mathbf{p}_{i}, \lambda_{i} \mathbf{f}_{e f}\right)$.

4. Check sign of $\tau_{i}$. If $\operatorname{sign}\left(\tau_{i}\right)<0$, setting $\mathbf{p}_{r}=\mathbf{p}_{i}$ and $\lambda_{r}=\lambda_{i}$, otherwise $\mathbf{p}_{l}=\mathbf{p}_{i}$ and $\lambda_{l}=\lambda_{i}$ and go back to step 1 .

The arc-length method proposed by Crisfield $[14,11]$ and previously discussed by the author in [3] is adopted to deal with the possible complex behavior in computing the

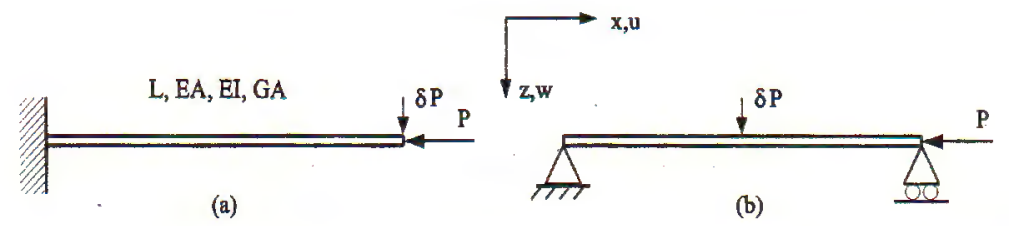

Fig. 4. Cantilever and simply supported beams under axial forces

equilibrium paths. In contrast with the critical load computation, it is necessary to introduce some appropriate perturbations, so that the secondary paths could be followed. 


\section{Numerical investigations}

The shear deformation in a beam becomes more important when the beam slenderness is lower. The slenderness of a beam can be defined through the parameter $s$, called slender parameter as

$$
s=\frac{L}{r}
$$

where $L$ is the total beam length, and $r$ is the gyration radius of the beam cross-section. Eq. (5.1) implies that for a higher value of the parameter $s$, more slender the beam is. That is the shear deformation will play a more important role for the beams having low value of the parameter $s$.

\subsection{The elastica}

\subsubsection{Element accuracy}

The accuracy of the developed element can be evaluated through the classical problems of cantilever (CF) and simply supported (SS) beams under axial forces as shown in Fig. 4. To perform numerical computation, the following geometrical and material data are adopted

$$
\begin{array}{ll}
L=5 \mathrm{~m}, \quad A=0.01 \mathrm{~m}^{2}, & I=1 \times 10^{-5} \mathrm{~m}^{4}, \\
E=2.1 \times 10^{11} \mathrm{~N} / \mathrm{m}^{2}, & \nu=0.3,
\end{array}
$$

where $L, A, I, E, \nu$ are the total length, cross-sectional area, moment of inertia, elastic modulus and Poisson ratio, respectively.

The beams with geometry and loading configurations in Fig. 4 exhibit 'the elastica phenomenon' with the analytical solutions given in $[15,16]$ The critical loads and equilibrium paths of the beams are computed herewith by employment of various element numbers. A shear correction factor $\psi=\frac{10(1+\nu)}{12+11 \nu}$ for rectangular cross-section [5], is adopted. For the purpose of comparison, a Bernoulli element based on the shallow arch theory denoted ' $b 2 B e^{\prime}$, previously formulated in [3], is also employed in the computation.

Table 1 lists the buckling coefficients $f_{c}$ of the CF and SS beams computed by the ' $62 T i$ ' and ' $b 2 B e^{\prime}$ ' elements and using different number of elements, where $f_{c}$ is defined as

$$
f_{c}=\frac{L^{2}}{E I} P_{c}
$$

with $P_{c}$ is the critical load.

Table 1. Buckling coefficients for CF and SS beams

\begin{tabular}{crrrr}
\hline & $\mathrm{CF}$ & \multicolumn{3}{c}{$\mathrm{SS}$} \\
$\mathrm{NE}^{*}$ & 'b2Ti' & 'b2Be' & 'b2Ti' & 'b2Be' \\
\hline 5 & 2.5080 & 2.4677 & 10.5481 & 9.8755 \\
10 & 2.4771 & 2.4676 & 10.0260 & 9.8736 \\
15 & 2.4714 & 2.4676 & 9.9341 & 9.8735 \\
20 & 2.4694 & 2.4676 & 9.9022 & 9.8734 \\
\hline
\end{tabular}

*number of elements 


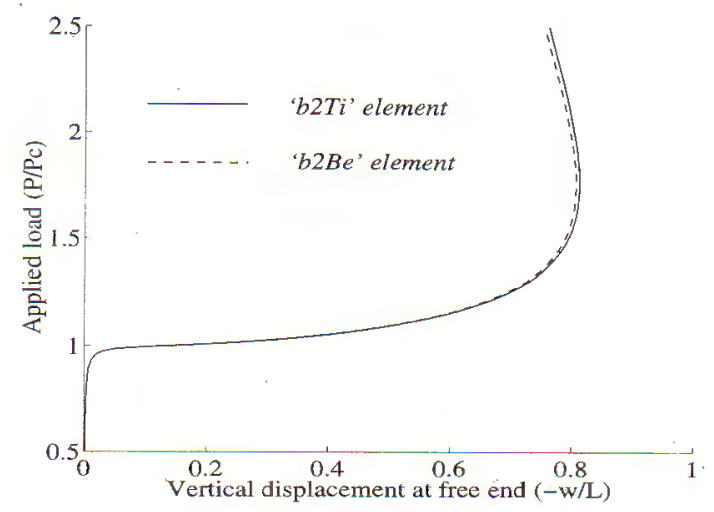

Fig.5. Equilibrium paths for CF beam computed by 4 elements of different types

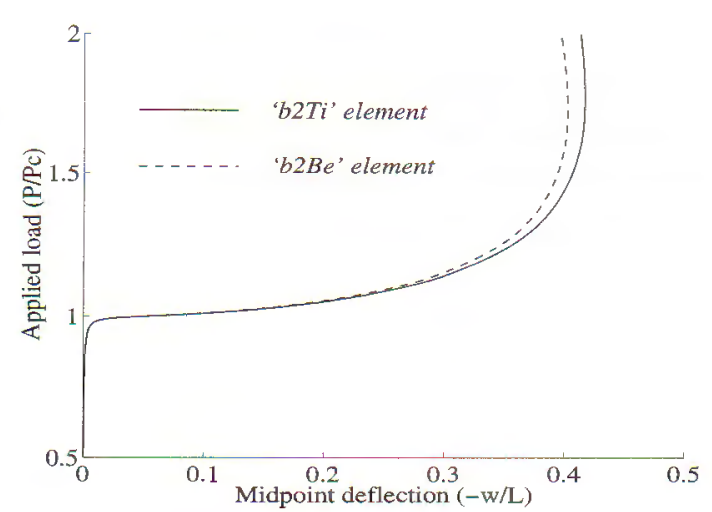

Fig. 6. Equilibrium paths for SS beam computed by by 4 elements of different types

The difference in the post-buckling paths for CF and SS beams computed by the

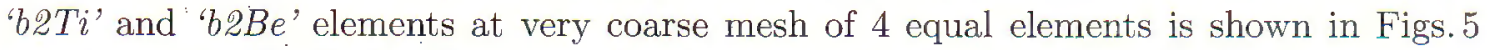
and 6 , respectively. As seen from the table and the figures, using a coarse mesh, the buckling coefficient and the post-buckling path computed by the ' $b 2 T i$ ' element is less accurate than that computed by the ' $62 B e^{\prime}$ ' element. The reason for this lies on the low order interpolation scheme used in formulating the ' $b 2 T i$ ' element. Comparing the linear functions adopted in the present work, the cubic polynomials employed in formulating

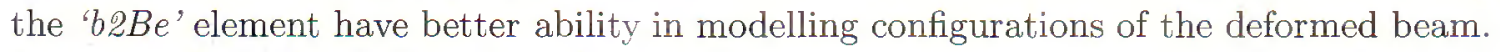
However, the difference between the critical loads computed by the two elements can be narrowed by refining the finite element mesh as seen from Table 1. Using a mesh of more that 6 elements, the difference in the post-buckling paths of the beams is almost invisible. In other words, the present formulated element is accurate in assessing the critical load and large displacement behavior, provided that a fine mesh is employed.

\subsubsection{Shear deformation modelling ability}

Table 2. Buckling coefficients at different slenderness

\begin{tabular}{ccccc}
\hline & \multicolumn{5}{c}{$s$} & \\
& 100 & 50 & 20 & 10 \\
\hline $\mathrm{CF}$ & 2.4683 & 2.4627 & 2.4244 & 2.2966 \\
$\mathrm{SS}$ & 9.8843 & 9.7954 & 9.2153 & 7.6066 \\
\hline
\end{tabular}

To investigate the ability of the developed element in modelling the shear deformation, the computation is performed for the beams having different values of the slender parameter $s$. Following the work in [4], we keep all the above data, except the cross-sectional area of the beam. In this regard, for $s=100,50,20$ and 10, the computation is carried out with the beam cross-sectional area $A=0.004,0.001,1.6^{-4}$ and $4^{-5} \mathrm{~m}^{2}$, respectively.

Table 2 gives the buckling coefficients of the CF and SS beams at different values of the slender parameter $s$. A mesh of 20 equal elements has been employed to compute the buckling coefficients in Table 2. As seen from Table 2, the buckling coefficient is 
gradually lowered with a reduction in value of the slender parameter $s$, regardless of the boundary conditions. The equilibrium paths of the beams at different slenderness displayed in Figs. 7 and 8 also show a clear influence of the shear deformation in the large displacement behavior of the beams. In other word, the 'b2Ti' element formulated in the present paper shows a good performance in modelling the effect of shear deformation.

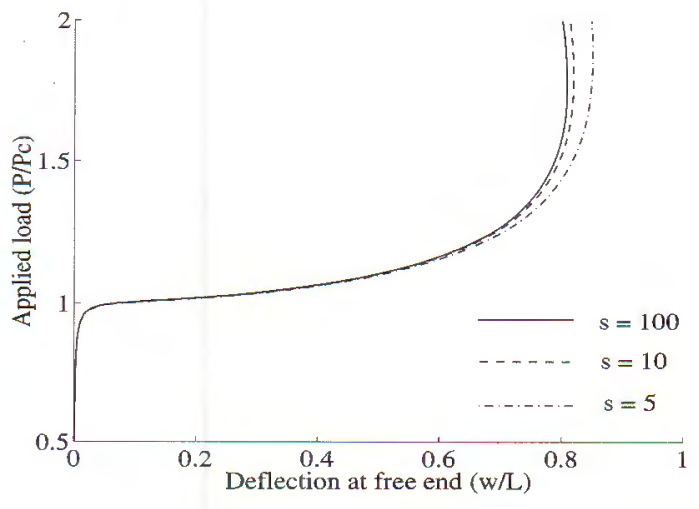

Fig. 7. Equilibrium paths for $\mathrm{CF}$ beam at different values of slender parameter

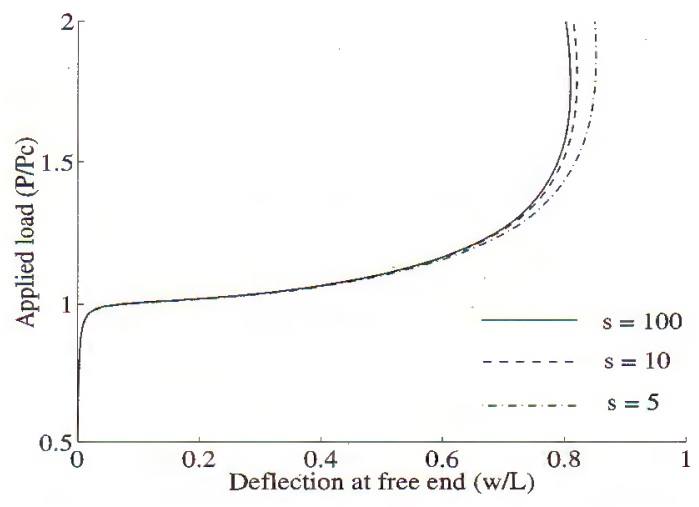

Fig. 8. Equilibrium paths for SS beam at different values of slender parameter

\subsection{Lee frame}

The asymmetric frame shown in Fig. 9 known in the literature as Lee frame, previously investigated analytically by Thompson and Hunt [17], and numerically by Hsiao and Huo [18].

The geometrical and material data of the frame are as follows:

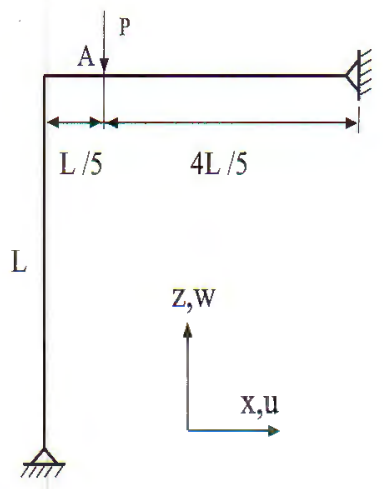

Fig. 9. Geometry configuration of Lee frame

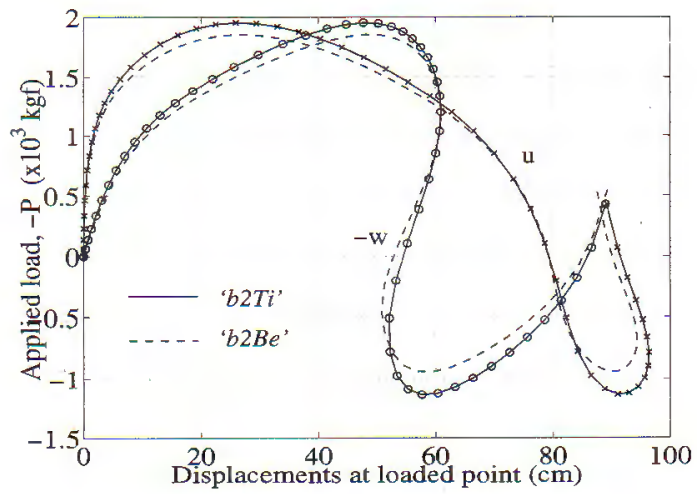

Fig. 10. Equilibrium paths for Lee frame

$$
\begin{aligned}
& L=120 \mathrm{~cm}, \quad A=6 \mathrm{~cm}^{2}, \quad I=2 \mathrm{~cm}^{4} \\
& E=7.2 \times 10^{5} \mathrm{kgf} / \mathrm{cm}^{2}, \quad G=1.69 \times 10^{5} \mathrm{kgf} / \mathrm{cm}^{2}
\end{aligned}
$$


The material data show that the frame is made of aluminum alloy. A concentrated load at point $A$ as shown in the figure is assumed.

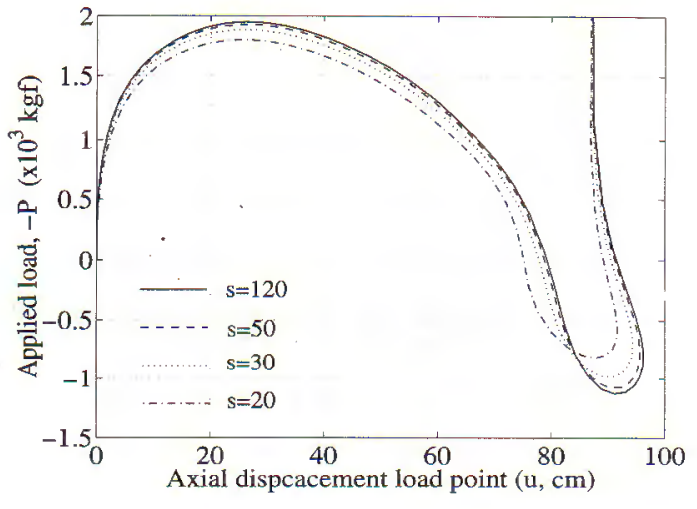

Fig. 11. Axial displacement versus applied load of Lee frame at different values of slenderness parameter

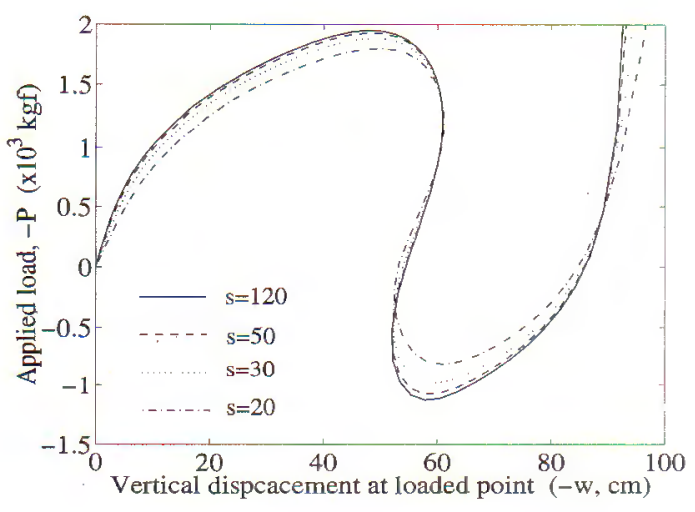

Fig. 12. Vertical displacement versus applied load of Lee frame at different values of slenderness parameter

Fig. 10 shows the equilibrium paths defined as the load versus the displacements at the

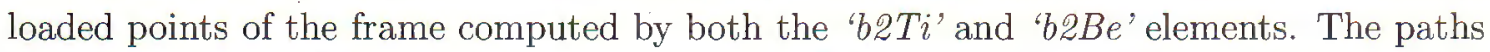
have been obtained by using 10 equal elements, 5 for each beam. The equilibrium paths displayed in Fig. 5.2 show that the ' $b 2 T i$ ' element is a bit stiffer than its counterpart, the ' $b 2 B e^{\prime}$ element. The reason for this, as explained above is the lower order interpolation scheme which has been employed in formulating the ' $62 T i$ ' element. The difference between the two elements can also be arrowed by increasing the number of elements in the analysis.

The axial and vertical displacements versus the applied load at various values of the slender parameter are given in Figs. 11 and 12, respectively. As in Sub-sec.5.1, the analysis is performed by keeping all the geometrical data excepts the cross-sectional area. The effect of shear deformation is again observed clearly from these Figures. The frame strength as well as its limit load are lower with a reduction in the slender parameter, and the numerical results show that the shear deformation must be taken into account for the frames constructed from stubby beams.

\subsection{Portable and multi-element frames}

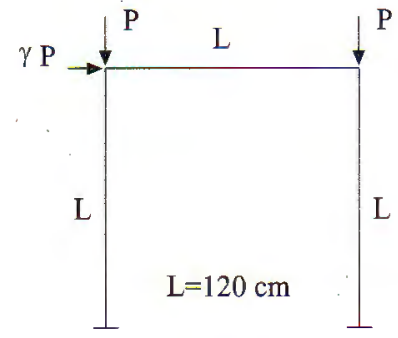

(a)

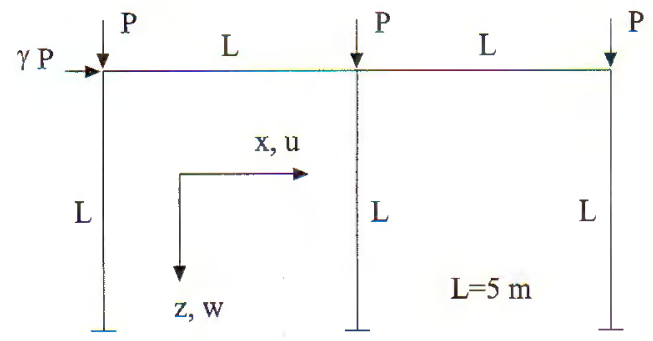

(b)

Fig. 13. A portable frame (a) and a multi-element frame (b) under concentrated loads 


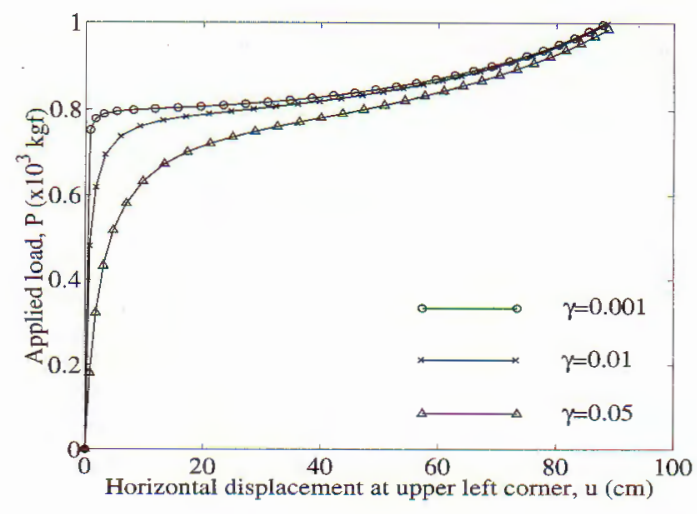

Fig. 14. Horizontal displacement versus applied load of portable frame at different values of axial force $\gamma P$

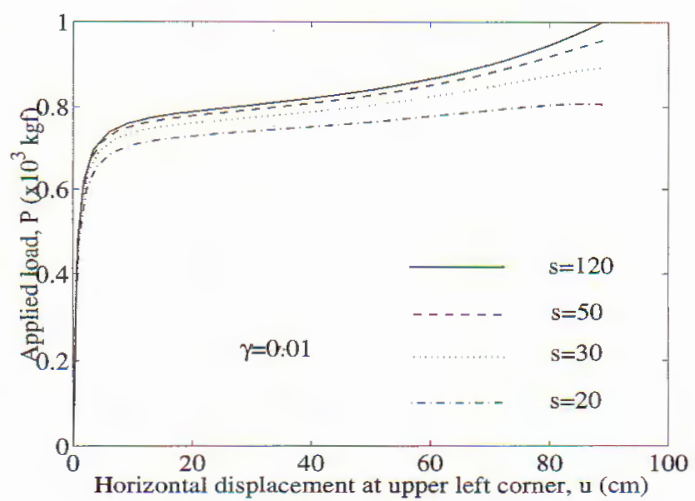

Fig. 16. Horizontal displacement versus applied load of portable frame at different values of slender parameter $s$

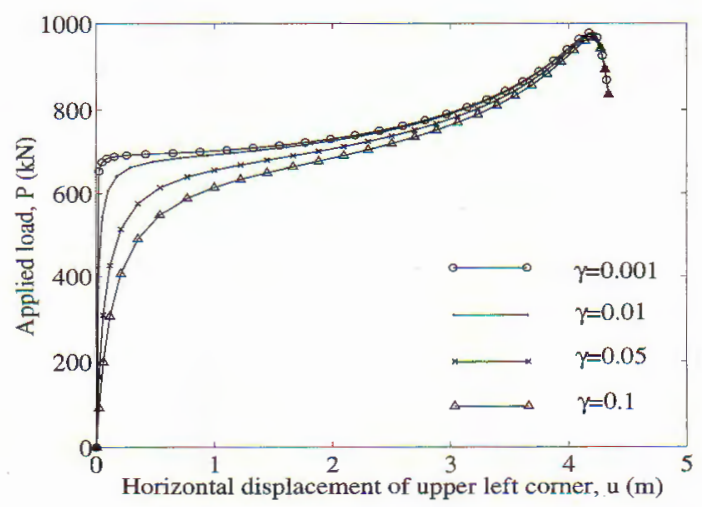

Fig. 18. Horizontal displacement versus applied load of multi-element steel frame at different values of axial force $\gamma P$

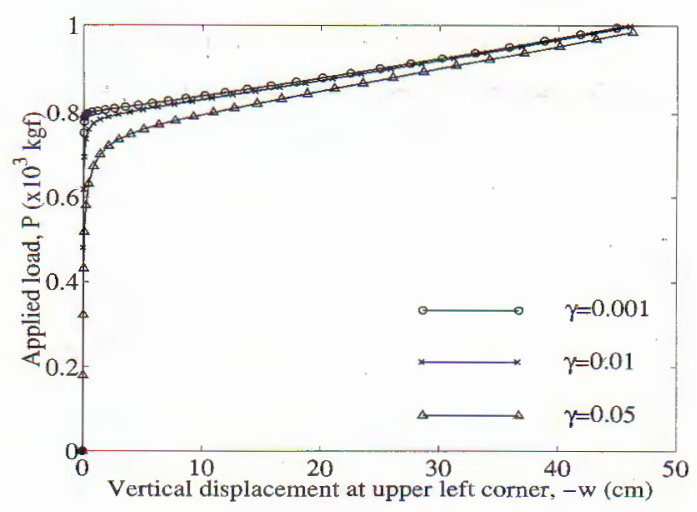

Fig. 15. Vertical displacement versus applied load of portable frame at different values of axial force $\gamma P$

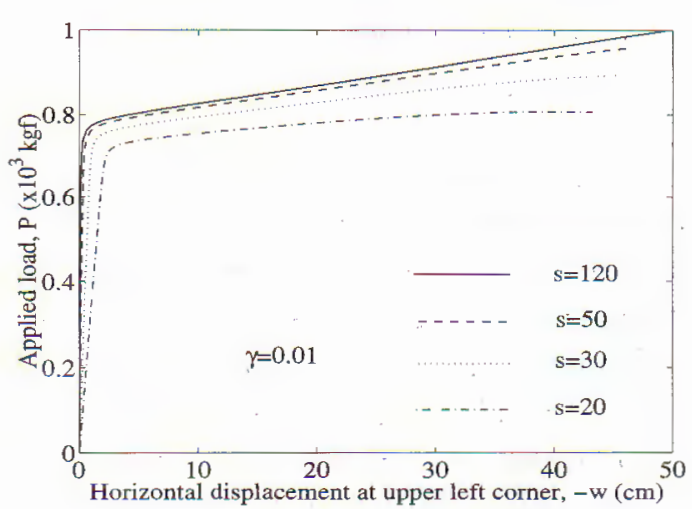

Fig. 17. Vertical displacement versus applied load of portable frame at different values of slender parameter $s$

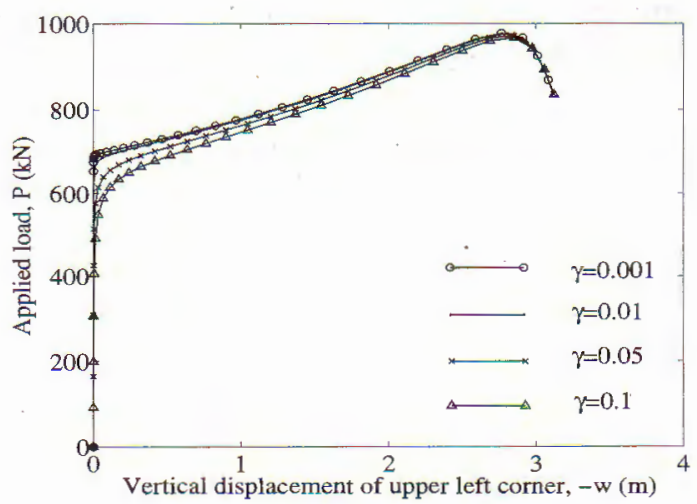

Fig. 19. Vertical displacement versus applied load of multi-element steel frame at different values of axial force $\gamma P$ 


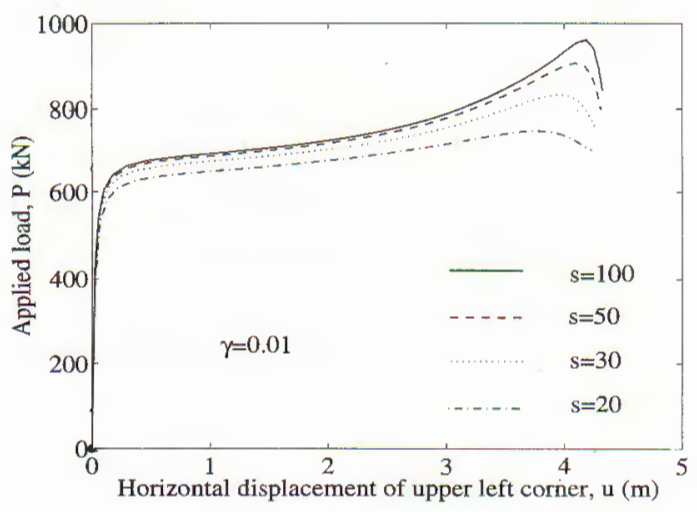

Fig. 20. Horizontal displacement versus applied load of multi-element steel frame at different values of slender parameter $s$

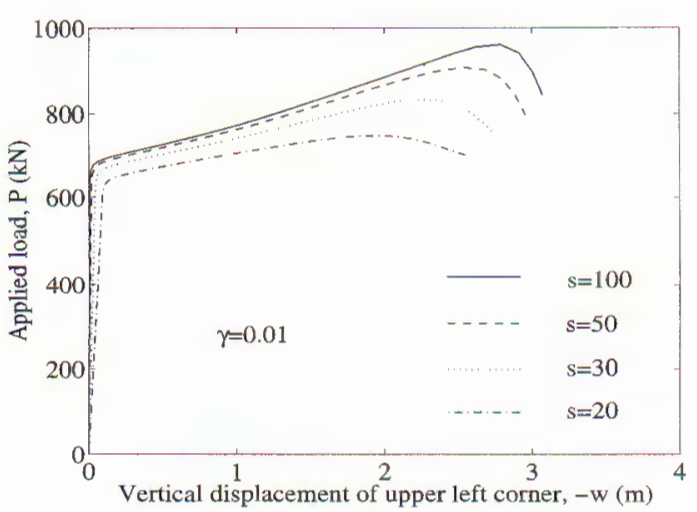

Fig. 21. Vertical displacement versus applied load of multi-element steel frame at different values of slender parameter $s$

A portable aluminum alloy frame and a multi-element steel frame under concentrated load as respectively shown in Fig. $13 \mathrm{a}$ and Fig. $13 \mathrm{~b}$ are analyzed in this sub-section. This sub-section aims to show the effects of the slenderness on the large displacement behavior of more practical structures. The geometrical and material data for the portable and multi-element frames are assumed the same as that of the Lee frame and the beams in Sub-secs.5.2 and 5.1, respectively.

The equilibrium paths, defined as the displacement versus applied load for the portable frame at different values of the axial force are given in Figs. 14 and 15. The paths computed with various values of the slenderness parameter are given in Figs. 16 and 17.

The computation has been performed by 12 equal ' $b 2 T i$ ' elements, 4 for each beam. Thus, the total number of active nodal displacement is 33 . The high number of elements employed in order to reduce the possible error, which the ' $62 T i$ ' element may produce as seen in Sub-sec.5.1. As seen from Figs. 14 and 15, the displacement-load curve of the frame much depends on the horizontal force, and the value of this force clearly affects the large displacement behavior of the frame. The strength measured in the large displacement region of the frame, as seen from Figs. 16 and 17, is reduced with a reduction in the slenderness parameter.

Figs. 18 and 19 show the equilibrium paths of the multi-element steel frame at various values of the axial force. The effects of slenderness on the large displacement behavior of the frame are displayed by Figs. 20 and 21. In general, the large displacement behavior of the present steel frame is similar to that of the aluminum alloy frame. The buckling load as well and the post-buckling strength of the frame are also reduced when lowering the slender parameter. The numerical results obtained in this sub-section again confirm the importance of shear deformation in the large displacement behavior of the frame structures, and we should not ignore it when analyzing frames forming from the elements having low slenderness. 


\section{Conclusions}

The large displacement analysis of elastic frame has been carried out in the present paper by the finite element method. Adopting the co-rotational approach, a two-node nonlinear element based on the Timoshenko beam theory has been formulated and employed to compute the critical loads and equilibrium paths of some frame structures. A detail investigation on the influence of the slenderness on the large displacement behavior of the frames has been performed. The main conclusions of the paper can be summarized as follows:

1. The element with shear deformation, formulated by employing the linear functions in interpolating the displacement field, needs a finer mesh to achieve the accuracy which the Bernoulli element does, but has good ability in modelling the shear effects.

2. The critical load and the post-buckling strength of the frame structures are affected by the slenderness, and they are smaller for the frames forming from low slenderness components. In other words, the large displacement behavior of the frames is affected by the shear deformation, and this deformation should be taken into account when the frame components having low slenderness.

\section{References}

1. Belytschko T., Liu W. K., Moran B., Nonlinear Finite Elements for Continua and Structures, John Wiley \& Sons, Chichester, 2000.

2. Nguyen D. K., "A non-linear element for analyzing elastic frame at large deflections", Vietnam Journal of Mechanics 22 (2000) 19-28.

3. Nguyen D. K., Do Q. Q., "Large deflections analysis of frames by elements contaning higer-order terms", Vietnam Journal of Mechanics 25 (2003) 243-254.

4. Nguyen D. K., "Post-buckling behavior of beam on two-parameter elastic foundation", International Journal of Structural Stability and Dynamics 42004 21-43.

5. Shames I. H., Dym C. L., Energy and Finite Element Methods in Structural Mechanics, McGraw-Hill, New York, 1989.

6. Cook R. D., Concepts and Applications of Finite Element Analysis, $4^{\text {th }}$ edition, John Wiley \& Sons, New York, 1989.

7. Hughes T. J. R., The Finite Element Method. Linear Static and Dynamic Finite Element Analysis, Dover publication, Inc., Mineola, 2000.

8. Reddy J. N., "On locking-free shear deformable beam finite elements", Computer Methods in Applied Mechanics and Engineering 149 (1997) 113-132.

9. Crisfield M. A., Finite Elements and Solution Procedures for Structural Analysis. Volume 1: Linear analysis, Pineridge Press, Swansea, 1986.

10 Owen D. R. J and Hinton E., Finite elements in plasticity: theory and practice, Pineridge Press, Swansea, U. K., 1980.

11 Crisfield M. A., Non-linear Finite Element Analysis of Solids and Structures, Volume 1: Essentials, John Wiley \& Sons, Chichester, 1991. 
12. Shi J., Computing critical points and secondary paths in nonlinear structural stability analysis by the finite element method, Computers \& Structures 58 (1996) 203-220.

13 Crisfield M. A., Non-linear Finite Element Analysis of Solids and Structures, Volume 2: Advanced topics, John Wiley \& Sons, Chichester, 1997.

14. Crisfield M. A., A fast incremental/iterative solution procedure that handles 'snapthrough', Computers \& Structures 13 (1981) 55-62.

15. Timoshenko S. P., Gere J. M., Theory of Elastic Stability, 2nd edition, McGraw-Hill, New York, 1961.

16. Dym C. L., Stability Theory and Its Applications to Structural Mechanics; Dover Publications, Inc., New York, 2002.

17. Thompson J. M. T., Hunt G. W., A General Theory of Elastic Stability, John Wiley \& Sons,London, 1973.

18. Hsiao K. M.,Huo F. Y.0, Nonlinear finite element analysis of elastic frames, Computers ES Structures 26 (1987) 693-701.

Received April 14, 2004

\section{ẢNH HƯỜNG CỦA BIẾN DANG TRƯợT TỚI ĐỐI XƯ CỦA KHUNG ĐÀN HỒI CÓ ĐỘ VÕNG LỚN}

Bài báo nghiên cứu ảnh hường của biến dạng trượt tới đối xử của khung đàn hồi có độ võng lớn. Phần tử dầm phi tuyến hai nút có tính tới ảnh hương của biến dạng trượt xây dựng trên cơ sở phương pháp năng lượng và kỹ thuật hệ tọa độ đồng hành được ứng dụng để phân tích các kết cấu khung. Giá trị lực tới hạn cưa kết cấu được tính bằng phương pháp khoanh vùng trong đó sử dụng giá trị riêng thấp nhất của ma trận độ cứng tiếp tuyến kết cấu làm tham số chì định. Đường cân bằng của kết cấu được xây dựng bằng phương pháp lặp tăng dần và kỹ thuật độ dài cung. Kết quả số chỉ ra rằng biến dạng trượt đóng vai trò quan trọng tới giá trị lực tới hạn và đối xừ khi có độ võng lớn của khung hình thành từ các dầm mảnh. Nghiên cứu chi tiết được thực hiện làm sáng tỏ ảnh hương của độ mảnh tới đối xử của khung có độ võng lớn. 